\title{
Theory of Stress-Anxiety-Sleep Disorders-Neural Damage Cyclic Chain and the Progression of Parkinson's Disease
}

\author{
Jorge Garza-Ulloa* \\ Research Consulting Services garzaulloa.org, University of Texas El Paso, USA
}

*Corresponding author: Jorge Garza-Ulloa, Research Consulting Services garzaulloa.org, University of Texas El Paso, USA.

To Cite This Article: Jorge Garza-Ulloa.Theory of Stress-Anxiety-Sleep Disorders-Neural Damage Cyclic Chain and the Progression of Parkinson's Disease. Am J Biomed Sci \& Res. 2019 - 6(3). AJBSR.MS.ID.001021. DOI: 10.34297/AJBSR.2019.06.001021.

Received: 泚 November 08, 2019; Published: 眥 November 19, 2019

\begin{abstract}
"Parkinson' disease" is a neurologic complex disease that affect the functionality if the brain modifying the neural connectivity due to cell death, that still don't have cure. All actual medication and procedures available today only help to improve the quality of life of the patients. The main objective of this research paper is to conclude a theory for the relation of "Stress", "Anxiety" and "Sleep disorders" and an additional factor identify as "neural damage", and relation between them and others additional factors like genetic disorders with the progression of "Parkinson's disease", supported with many published research papers that are currently available, personal interviews with PD patients, caregivers, PD families, neurologists, others peoples involves in their PD health team, and my own experience as a Biomedical Engineering researcher and caregiver for my wife with PD.

"The Theory of Stress-Anxiety-Sleep Disorders-Neural Damage cyclic chain and the progression of Parkinson's disease ", it is explained with concept involved, many reasons to be included in the theory and their relations to previous conclusions/suggestions of many researchers papers already publish, and recommendations to develop good habits in PD patients, with correct attitude and actions to implement the theory to help in the goal of achieve a decrease in the progression of Parkinson's disease. They are concentrated as "The 7 most important recommendations for PD patients and their caregivers"
\end{abstract}

Keywords: Parkinson's Disease; Tremors; Movement Disorders; Postural Instability; Stress; Anxiety; Sleep Disorders; Neuronal Damage; Neurons; Neuronal Pathways; Parkinsonism; Progressive Rapid Eye Movements.

\section{Introduction}

\section{Stress-Anxiety-Sleep Disorders -Neural Damage Chain, Parkinsonism and Parkinson's Disease}

"Parkinsonism" is a constellation of signs and symptoms that are characteristically observed in "Parkinson's disease (PD)", but that are not necessarily due to PD. Parkinsonism is the primary type of hypokinetic movement disorder. Parkinsonism main symptoms are generally classified of three kinds [1]: "primary motors", "secondary motors" and "non-motors". Where:

1. "Primary motors" are directly related to movement disorders as: tremors in the limbs, muscles stiffness or rigidity, bradykinesia or slowness of movements, postural instability as difficult on standing/walking/balance, etc.

2. "Secondary motors" are consequence of movement disorders as: hypomimia or loss of facial expressions, freezing of gait or shuffling gait, speech difficulty or changes in speech, stooped posture, dystonia or prolonged muscle contractions, repetitive movement and many others.

3. "non-motors" that are not related to movement disorders as: cognitive decline, sleep problems, fatigue or excessive tiredness that isn't relieved with sleep, pain which can be accompany of muscle rigidity, mood changes including: apathy, depression, anxiety and involuntary/ uncontrollable outbursts of crying or laughing, and many others [2].

The main objective of this research is focus on four factors that form a kind of a chain connection between them, they are: "StressAnxiety-Sleep disorders-neural damage cyclic chain that affect the progression of Parkinson's disease", the initial factor could be any 
of the four factors or other that are incorporated in them to behave as a reaction chain. Where:

a. "Stress" is defined as a physical, mental, or emotional factor that causes bodily or mental tension. Stress can be external (from the environment, psychological, or social situations) or internal (illness, pain or from a medical procedure).

b. "Anxiety" is an emotion characterized by feelings of tension, worried thoughts that lead to excessive nervousness, fear, apprehension, and inclusive physical symptoms changes as increased blood pressure and others that may seriously affect day-to-day living.

c. "Sleep disorders" are frequently initiated with "sleep changes patterns" due to a big diversity of reasons as: "stress", "anxiety", aging, hormonal levels changes, mood, sleep apnea, snoring, lifestyles, environment changes, restless leg syndrome, and many other reasons. Sleep is defined as the normal condition of body and mind such as that which typically recurs for several hours every night, in which the nervous system is relatively inactive, the eyes closed, the postural muscles relaxed with consciousness practically suspended and the normal "Sleep stages "are four, where duration is different at various age [3]:

i. Stage one: "Sleep" begins with a "lighter sleep" that can last from seconds up to seven minutes like a "short nap" where we can be easily woken, while the brain produces "alpha" and "theta" waves, and your eye movements slow down.

ii. Stage two: It is also a similar to a "light sleep" resembling a "larger nap", where we can wake up if we want. Here the brain produces a sudden increase in brain waves known as "sleep spindles", then the brain waves slow down.

iii. Stage three: It is the beginning of a "deep sleep" and becomes little harder to wake up because the body became less responsive to external stimulus. Here the brain begins producing slower "delta waves" and it is characterized by "no movement active on the eyes or muscles".

iv. Stage four: It move to a "deeper sleep", and it is harder to wake up. Here the brain is more active producing more "delta waves" allowing a necessary "restorative stage", where the body repairs muscles, tissues, tiny bone fractures, diminish pain, stimulates growth and development, boosts immune function, and builds up energy for the next day. About 90 minutes after initially falling sleep a "Rapid Eye Movement [REM)" is observed, were the eyes makes sudden movements in different directions, heart rate and blood pressure increase, and breathing becomes fast, irregular, and shallow. "REM" can last up to an hour and an adult can have five or six of these cycles with intervals :"REM" and "non-rapid eye movement (NREM " known a "progressive Rapid Eye Movements (pREM)" [4], where the brain consolidates and processes information from the day before so that it can be stored in your long-term memory.

The "Stress", "Anxiety" and "Sleep disorders" have a profound effect on a person's, overall health and they are deeply connected between them. In summary, we can say that the three factors are related as a response, reaction and action chain effect [5], as follow:

a. "Stress" is a response to a threat in a situation,

b. "Anxiety" is a reaction to the "stress", and

c. "Sleep disorders" is the action effect of "stress" and/ or "anxiety" and others factors as: aging, hormonal levels changes, mood, sleep apnea, snoring, lifestyles, environment changes, restless leg syndrome, etc.,

"Parkinson's disease (PD)" patients present "Stress-AnxietySleep disorders" affecting their "sleep cycles in quality and duration, especially in stage four while being in pREM", this alteration is known as "REM Behavior Disorders" is characterized by "dream enactment (acting while dreaming)", insomnia, sleep apnea, and others factors, and is associated with incidence on their "neurodegenerative disorders", where the muscle paralysis that normally occurs during "REM sleep" is incomplete or absent [6]

\section{Understanding the Relation of Human Nervous System, Neurons, Neural Pathways, Neurotransmitters and Parkinson's Disease}

It is very important to understand the concepts of how the "human nervous system", "neurons", "neural pathways", "synapses" and "neurotransmitters" work together in the human body, to find the relation of many neurologic diseases as: Parkinson's disease, Multiple System Atrophy, Lewy body dementia (Lewy bodies), Alzheimer's disease, Huntington's disease, and others. They could make use of the proposed cyclic chain "Stress-Anxiety-Sleep disorders, Neural damage" for their progression. The concepts can be explained as follow [7]:

A. "Human nervous system". It is the system of the human body that coordinate a person's voluntary and involuntary action, and transmit signals between different part of the body, it consists of two parts as shown in Figure 1a: "Central Nervous System (CNS) that is integrated by the brain and spinal cord" and "peripheral nervous system that consists of mainly nerves". The primary function of the human nervous system is to control the body through a complex collection of nerves and specialized cells known as "neurons or nerve cells". 
B. "Neuron" is a specialized cell known as the basic unit of the "human nervous system". These cells make a up a massive network of "specialized nerve cells" connected with the purpose of transmit messages very rapidly, from one part of the body to another, creating complex interconnected circuits known as: "neural pathways". A zoomed illustration of two neurons connected are shown in Figure 1b, to introduce the concept of "Neural Pathways".

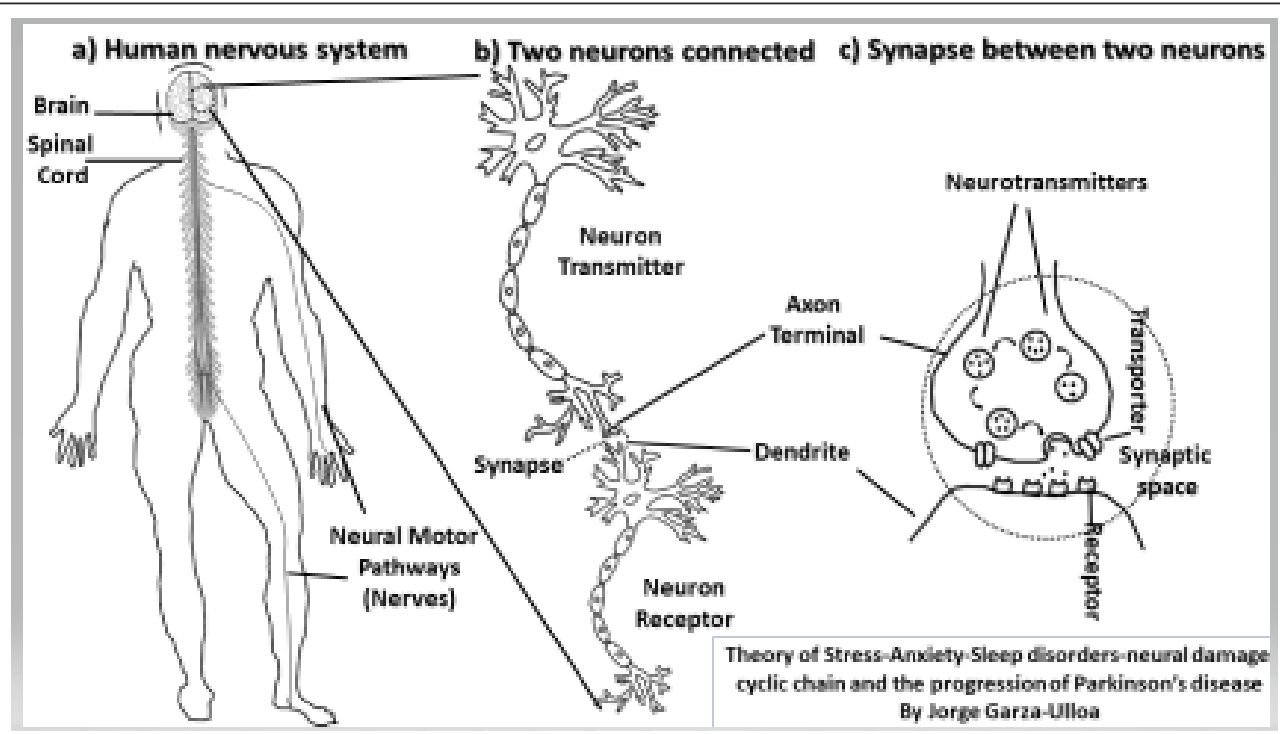

Figure 1: The relation between human nervous system, neurons, neural pathways, and synapse: a) Human Nervous System and two Neural Pathways, 2) Two neurons connected as a part of the Neural Pathway in the brain, and c) Synapse between two neurons that are part of a Neural Pathway.

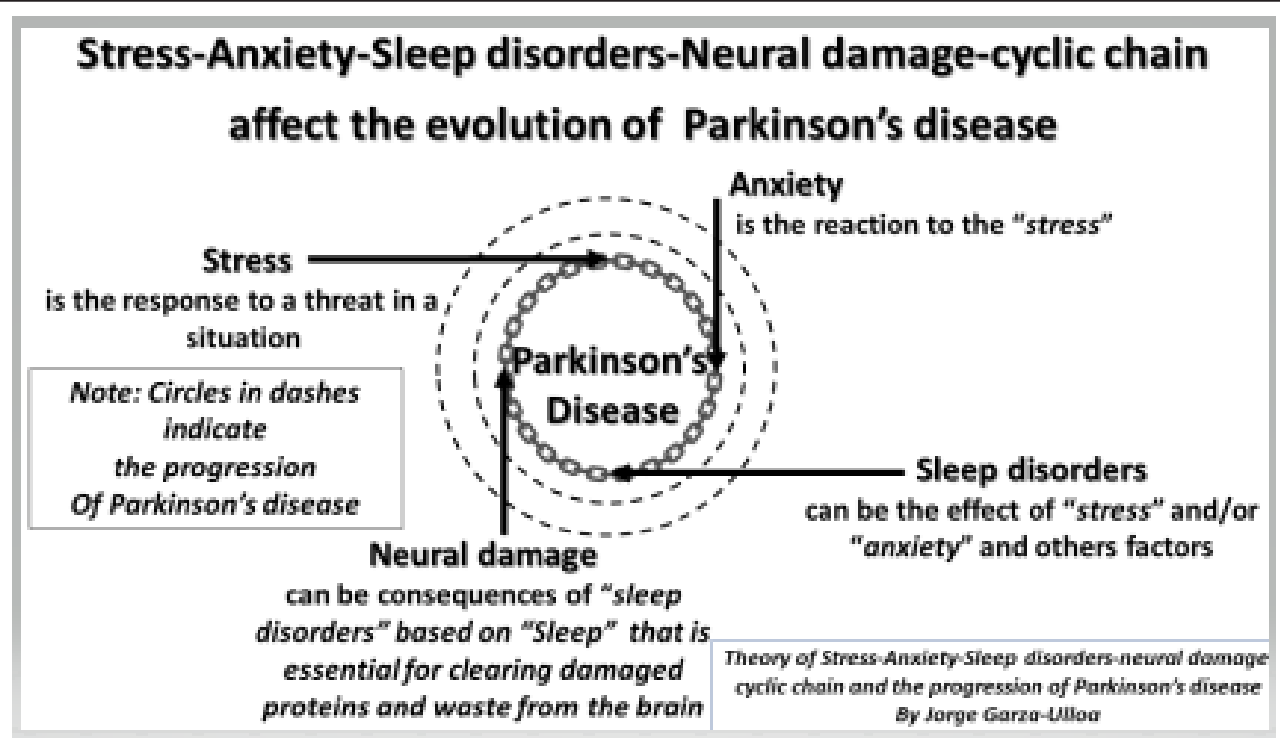

Figure 2: Stress-Anxiety-Sleep Disorders-Neural Damage cyclic chain and Parkinson's disease.

C. "Neural pathway" is a cluster of "neurons" connection from one part of the nervous system to another using "synapses" in each neurons union. The brain under the right conditions can change hundreds of millions and possibly billions of the connections between the nerve cells in our "neural pathways" as we learn, and process new information [8]. The "Neural pathways" created in the brain are updated continuously based on "neurons" connection process known as "synapse", there are an estimated of 100 billon neurons making a total of 100 trillion neural connections, that process information and they can send orders to body movement known as "motor pathways", and receive information from humans senses known as "sensory pathways".

D. "Synapse" is the structure that permits a "neuron" to pass an electrical to chemical signal from one neuron to another. The "synapse" is located in the union between two "neurons" that are part of a "neural pathway", as indicated in Figure 1c zooming of a "synapse" where, a 
"neuron" is connected through the "Axon Terminal of the Neuron Transmitter" to another "neuron" through the "Dendrite of the Neuron Receptor".

"Neurons basic functions" can be categorized as Sensory, Motor and Interneurons. Where:

a. "Sensory that carry information from the sensory receptor cells as eyes, nose, tongue, and skin throughout the body to the brain",

b. "Motor that transmit information from the brain and spinal cord to the muscles of the body to achieve movements on them", and

c. "Interneurons that convey information by transmitting messages to other neurons".

"Neuron activities" are of two kinds Electrical and Chemical. Where:

a. "Electrical that is used to transmit signals within neurons", and

b. "Chemical that is used to transmit signal between neurons through the synaptic space".

The "synapse chemical activity" trigger the release of "neurotransmitters" which carry the impulse across the synapse to the next neuron. Once a nerve impulse has triggered the release of "neurotransmitter", these chemical messengers cross the tiny "synaptic space" and taken up by specialized receptors on the surface of the next cell. "This process converts the chemical signal back into electrical signal", if the signal is strong enough, it will be propagated down to the next neuron until once again reaches another "synapse" and the process is repeated once more, and so on until reach the destination.

The "neurotransmitters" than cross the "synapse" as indicated in Figure 1c are received by other nerve cells, where the "axon terminal from the neuron transmitter" is also responsible for the reuptake of any excessive "neurotransmitters" released during this process. Different "neurotransmitters" fulfill different functions in the brain as: "excitatory neurotransmitters" that acts to stimulate the firing in the "synapse", and "inhibitory neurotransmitters" that tend to block it in the responding cell. There are different types of small molecules manufactured in different kind of axon terminals, known generally as "neurotransmitters", some of them are: "acetylcholine", "dopamine", “GABA"," glutamate", "glycine”, "norepinephrine", "epinephrine or adrenaline", "serotonin", and many more. Where [7]:
1. "Acetylcholine (Ach)" is used by spinal cord motor neurons to cause muscle contraction, and many neurons in the brain to regulate memory. In most instances, "acetylcholine" is excitatory function.

2. "Dopamine (DA)" is responsible for transmitting signals between the nerve cells in the brain. The brain includes several distinct "neural dopamine pathways" that play a major role in regulate movement, attention, learning, emotional responses the motivational component of reward-motivated behavior and also play a role in addiction. "Dopamine" functions as both "inhibitory and excitatory neurotransmitter" depending upon where in the brain and at which receptor site it binds to.

3. "GABA or (Gamma-aminobutyric acid) "is the major "inhibitory neurotransmitter" in the brain. It is important in producing sleep, reducing anxiety, and forming memory.

4. "Glutamate (Glu)" is the most "excitatory neurotransmitter" in the brain. It is important in learning and memory.

5. "Glycine (Gly)" is used mainly by "neurons" in the "spinal cord". It is probably always act as "inhibitory neurotransmitter".

6. "Norepinephrine (NE)" acts as a "neurotransmitter and a hormone". In the peripherical nervous system, it is part of the "fight-or-flight response", and in the brain, it acts a "neurotransmitter" regulating blood pressure and calmness. NE is usually excitatory, but it is inhibitory in a few brain areas.

7. "Epinephrine or adrenaline" is also a hormone responsible for concentration, attention, mood and both physical and mental arousal

8. "Serotonin (5-HT)" is involved in many functions including mood, appetite, and sensory perception. In the spinal cord, serotonin is inhibitory in pain.

9. And many more additional "neurotransmitters", where the action of all of them help to regulate many human body functions of different types.

It is very import to notice that each "neurotransmitter" work alone or with others to handle a big diversity of body functions that affect the three types of "Parkinson's symptoms" as indicated in Table 1.

Table 1: Some of the "Neurotransmitter" released in the "Synapses", their job is to regulates human functions. If their release is affected, their effect could be observed as Parkinson's symptoms.

\begin{tabular}{|c|c|c|}
\hline $\begin{array}{c}\text { Neurotransmitter } \\
\text { release in Synapses }\end{array}$ & Human body function & Parkinson's symptoms \\
\hline Acetylcholine & Regulate memory & "non-motors" \\
\hline
\end{tabular}




\begin{tabular}{|c|c|c|}
\hline Dopamine & $\begin{array}{l}\text { Regulate movement, attention, learning, } \\
\text { emotional responses and addiction }\end{array}$ & $\begin{array}{l}\text { "Primary motors", } \\
\text { "Secondary motors" } \\
\text { and "non-motors" }\end{array}$ \\
\hline GABA & $\begin{array}{l}\text { Producing sleep, reducing anxiety, and forming } \\
\text { memory }\end{array}$ & "non-motors" \\
\hline Glutamate & Handle learning and memory & "non-motors" \\
\hline Norepinephrine & Regulating blood pressure and calmness & $\begin{array}{c}\text { "Secondary motors" } \\
\text { "non-motors" }\end{array}$ \\
\hline Epinephrine commonly known as adrenaline & $\begin{array}{l}\text { Responsible for concentration, attention, mood } \\
\text { and both physical and mental arousal }\end{array}$ & "non-motors" \\
\hline Serotonin & $\begin{array}{l}\text { Regulating mood, appetite, and sensory } \\
\text { perception, and inhibitory of pain }\end{array}$ & "non-motors" \\
\hline
\end{tabular}

Research Papers that Support Factors that Generate PD Symptoms as "Stress-Anxiety-Sleep Disorders-Neural

\section{Damage"}

A general search of results of different investigations at the scientific publications that conclude or suggest part of the interaction between "Stress-Anxiety-Sleep Disorders-Neural damage cyclic chain and Parkinson's disease", some of them are:

\section{“Sleep Disorders":}

i. "Sleep disturbance" is a major non-motor complaint of "Parkinson's disease" with extensive impact on patient quality of life. Despite the overwhelming prevalence of this symptom, the impact of PD-related sleep disturbance is often underappreciated in light of the profound motor disorder experienced by this population. Current estimates of PDrelated "sleep disturbance" indicate that up to $98 \%$ of PD patients exhibit some symptoms of "sleep disturbance", often with significant daytime impairment reported in the research paper of “T.J. et al. Swick in the Parkinson's disease and sleep/ wake disturbances" $[8,9]$.

ii. "Parkinson's disease" patients report "disturbed sleep patterns long before motor dysfunction" in the research paper from "Jorge S.Valadas et al. ER Lipid Defects in Neuropeptidergic Neurons Impair Sleep Patterns in Parkinson's Disease" [10].

\section{"Neural damage" and "Sleep Disorders":}

i. "Sleep disorders" are frequent in "Parkinson's disease (PD)". Cyclic alternating pattern (CAP) constitutes an "NREM sleep instability marker". NREM sleep instability is present even in earlier stages of Parkinson's disease. The main result of our study consists in the disclosure of altered NREM sleep microstructure in PD even at an early stage of the disease, suggesting an "early alteration of the central pathways" involved in the NREM sleep building-up and stability, reported by "Lorenzo Priano et al. Sleep microstructure in Parkinson's disease: cycling alternating pattern (CAP) as a sensitive marker of early NREM sleep instability" [11]. ii. "Sleep loss" affects many aspects of cognition, and memory consolidation processes occurring in the brain specially in the "hippocampus" that seem particularly vulnerable to sleep loss, from the research paper of "James E. Delorme et.al. Sleep loss disrupts Arc expression in dentate gyrus neurons" [12].

iii. "Parkinson's disease" is the most common movement disorder in the broad spectrum of neurodegenerative diseases, associated frequently with gradual decline of the higher mental faculties. From the morphological point of view, "it is characterized by the degeneration of a substantial number of dopaminergic neurons of the substantia nigra and a considerable degeneration of neuronal networks in locus coeruleus, putamen, globus pallidus, thalamus and some areas of the cortex of the brain hemispheres". Filamentous inclusions, in the form of "Lewy bodies and Lewy neuritis", composed mainly of "alpha synuclein", been the hallmark of diffuse "Lewy body dementia", have been described in the neurons of the substantia nigra in many cases of "Parkinson's disease" associated with dementia. In previous studies we have described the morphological alterations in the "synapses" in the "caudate nucleus" and the "globus pallidus" in cases of "Parkinson's disease". The majority of the "synapses demonstrated changes in size and shape of the pre- and postsynaptic components, polymorphism of the synaptic vesicles and marked morphological alterations of the mitochondria". The morphological alterations of the "synapses" in cases of "Parkinson's disease" associated with dementia, plead in favor of the importance of the "neuronal circuits of locus coeruleus in cognitive functions". From research paper of "Stavros J Baloyannis, et al. Morphological alterations of the synapses in the locus coeruleus in Parkinson's disease" [13].

\section{"Anxiety" and "Sleep disorders":}

"Sleep Disturbances" are a non-motor symptom very common in "Parkinson's Disease" characterized by insomnia, worse quality of sleep and excessive daytime sleepiness. Sleep problems, excessive daytime sleepiness, depressive and "anxiety" symptoms are frequent in PD patients and have significantly associated each 
other, from "Rosanna Palmeri, Potential predictors of quality of life in Parkinson's Disease: Sleep and mood disorders" [14].

\section{"PD faster progression", "PD cognitive symptoms progression"} and "Sleep disorders":

"Rapid Eye Movement (REM) sleep behavior disorder (RBD) "is characterized by dream enactment and is associated with incidence of neurodegenerative disorders, especially "Parkinson's disease (PD)". Whether PD with RBD constitutes a distinct subtype with unique progression is unknown. Here, we investigated motor and cognitive symptom progression in patients with self-reported RBD features in adult life. Ours is a large population-based study with movement disorder specialist confirmed PD diagnoses and motor assessments. We present evidence that pRBD features may be an early clinical marker of faster cognitive decline and progression of motor symptoms in PD, the latter particularly for patients with marked Instability and Gait Dysfunction (PIGD) symptoms early in the disease. RBD-features may be a simple and useful screening for treatment trials and in clinical practice to identify those at risk for faster progression, who may benefit from pharmacological (changes in drug schemes) and non-pharmacological (including physical activity and prevention of falls)" interventions. From research paper of "AlineDuarte Folle et al. Clinical progression in Parkinson's disease with features of REM sleep behavior disorder: A population-based longitudinal study" [15].

\section{Progression of PD}

Parkinson's progression is usually quite gradual as it is a progressive illness, with symptoms slowly growing worse over time. Many people with Parkinson's believe they had the condition for some time - often two to three years - before they look for a formal diagnosis. Often it is only when symptoms become obvious or start to interfere with daily life that people visit the doctor. Symptoms and responses to treatment vary from person to person, so it is not possible to accurately predict how Parkinson's will progress. For some people it may take many years for the condition to develop, for others it may take less time [16].

\section{Types of Parkinson's}

Based on "Parkinson's disease" is a typical movement disorder [17] and the 2nd most common neurodegenerative condition after "Alzheimer's disease". PD is a continuous neurological disorder where the symptoms continue to worsen gradually [18]. PD is a highly variable disease, meaning that different patients have different combination of symptoms, and those symptoms can be at varying severity levels. There are basically two general "Parkinson's types": "Idiopathic Parkinson's" and "atypical parkinsonism" [19]. Where:

a. "Idiopathic Parkinson's" because of their unknown source. It is the most common form of "Parkinsonism". It is a tremor predominant disorder that involves shaking and trembling. About $85 \%$ of people with parkinsonism have "idiopathic Parkinson's". This type of "Parkinson's disease" can begin at an earlier age but progresses more slowly. It has a lower risk of cognitive (brain function) decline, but the tremors may be more difficult to treat than other symptoms [20].

b. "Atypical parkinsonism" is less common; it is an instability and gait disorder that present more trouble with walking and balance. About 15\% of people with parkinsonism have Atypical parkinsonism disorders, these are rarer conditions and more difficult to treat. This type of Parkinson's disease happens at an older age but tends to progress quickly. Although people may experience fewer tremors or no tremors at all, they have a higher risk of cognitive decline.

\section{Factors for risk of Parkinson's disease}

Nobody know the specific cause for "Parkinson's diseases" except that about $20 \%$ are presumed to be "genetic variations" in the genetic combination of certain genes elevate the risk of PD. Studies have reported that mutation in the $[21,22]$ :

a. "LRRK2 (leucine-rich repeat kinase2)"

b. " PARK7 (Parkinsonism Associated Deglycase)",

c. "PRKN (Parkin RBRE3 Ubiquitin Protein Ligase)",

d. "PINK1 (PTEN-induced putative kinase1)", or "SNCA (alpha-synuclein) "contribute to the risk of PD [23].

Generally, scientists speculate that the interaction between gene mutations and environmental exposures can contribute to PD progression. Studies have listed few modifiable risk factors for $\mathrm{PD}$, the following factors are considered as some of the causative factors of PD [24,25] are: Exposure to pesticides, herbicides, some solvents, well-water drinking, metals as manganese, calcium intake, age, gender, and many more factors [1]. The cause of idiopathic Parkinson's disease has been hypothesized to involve an external agent, for example a pathogen, and one potential route of entry for such an agent could be via the gastrointestinal system. The interactions of gut microbes and the central nervous system, also known as the microbiota-gut-brain axis, have recently become a topic of intense research. The consistent differences in gut microbiota between Parkinson's patients and control subjects could lead to new diagnostic or therapeutic modalities

\section{The Stress-Anxiety-Sleep Disorders-Neural Damage Cyclic Chain and Parkinson's disease}

Keeping track of PD studies and their results in many research papers on neurologic diseases, many of them frequently recognized factors in the PD progression are: "stress", "anxiety", "sleep disorders", "neural damages" and others that can be reflected on them. Analyzing many of the current research papers, we can deduct that : "these factors are related and they are repetitive with 
behavior as a kind of cyclic chain, where the initial factor could be anyone of them and grow a little more the intensity on each loop affecting the "progression of Parkinson' disease" that can be evaluated as a level increased on the PD symptoms [26,27] and others way, and this phenomena can be represented as indicated in Figure 2.

It can be explained as follow:

"Stress" is defined as a normal feeling of response to an emotional or physical tension, and it is very frequently on "PD patients". Their body's reaction to the everyday challenges or demand in actions that has been altered, when patient's thing, feel or affront their physical and cognitive limitation that are diminished for the PD. Also, it could come from any event as "thoughts that increase frustration", "angry", "pain" or "nervousness". The most common symptoms for "PD stress" are:

Excessive worries in your mind producing physical effects as:
a. Rapid heart rate,
b. Sweaty palms or feet,
c. Feeling light-headed, faint, or dizzy,
d. And many more physical effects.

Feeling overwhelmed presenting mental situations as:
a. Poor concentration,
b. Forgetfulness,
c. Loss of inters in the world around them, and duties that must do,

d. and many others mental effects.

"Stress on PD patients have many reasons", but the biggest one is usually due to the overwhelm feeling of losing control of their bodies, being each time more dependable of others persons, that they need to take control of the situations and knowing before that PD is a neurodegenerative disorder and still there is not cure for it. Additional thinking in them generate an immense stress on them, deducting that their PD could affect even more their physical body functions and present mental issues, and the increase of their disabilities.

There is enough evidence that "stress rise the level of PD symptoms" as "tremors", "cognitive decline", "mood", "behavior", and many PD symptoms [14]. The reality is that "stress" make for them the situation worst to deal with it.

"Anxiety" is the normal reaction to stress, but in PD patients it is more detectable by physical effects and mental situation that are on the next level, as:

Difficulty of controlling worries, showing an exaggerated feeling for sudden shock or alarm producing physical effects and repetitive actions of: a. Aches, pains, tense muscles, gastrointestinal problems, chest pain and rapid heartbeat, dry mouth and difficulty swallowing, clenched jaw and grinding teeth.

b. Immune body systems affected showing frequently, allergies and infections [28].

c. Nervousness and shaking, ringing in the ear, nail biting, bigger involuntary body limbs movements, and pacing.

d. Increased need for use of drugs, drink alcohol, or smoke cigarettes.

Feeling almost defeat and presenting mental situations in the next level as:

a. Impossible concentrating by mind going blank or by quieting their mind, with racing thoughts, and panic.

b. Difficulty relaxing and presenting poor judgment in decisions.

c. Showing easy fatigue due to irritability, pain that cannot explain where, or due to muscle tension.

d. Detecting that "multitasking" and "multiprocessing "as ability for doing more of one task at once, or thinking many things is being damage or lost in PD.

e. Perceiving that the "planning" of action to do is each time more difficult to program and follow in PD.

There is enough evidence that "stress and anxiety increase faster the level of PD symptoms" as "tremors", "cognitive decline", "mood", "behavior", and many others PD symptoms [29]. The reality is that "Stress and Anxiety" make for them the situation worse and almost impossible to deal by themselves.

"Sleep disorders" are conditions that result in sleep changes that are affecting the overall health, safety and quality of life. Research has shown the effects of "poor sleep", such as reduced mood and concentration as well as increasing the risk of serious conditions such as obesity, heart disease, diabetes and other neurological diseases as PD.

"The theory that sleep is necessary to reset connections between brain cells and process information from the previous day is gaining credibility trough time as the results of many research and clinical trial are obtained, and now the observation in many neurological diseases are suggesting that sleep is essential for clearing damaged proteins and waste from the brain". This insight could lead to new ways of treating a range of diseases that may be linked to "Stress-Anxiety-Sleep disorders-neural damage cyclic chain" such as "Parkinson's diseases", "Alzheimer's disease", and many others neurologic diseases.

The more common "sleep disorders" are:

a) "Sleep apnea" as abnormal sleep patterns in breathing. 
It is important to mention that there are several types of sleep apnea.

b) "Restless legs syndrome (RLS)" also known as "WillisEkbom disease", causes an uncomfortable sensation and an urge to move the legs while trying to sleep.

c) "Narcolepsy" is a condition characterized by extreme sleepiness during the day and falling asleep suddenly during the day.

d) "Insomnia" is characterized by having difficult to falling sleep or staying asleep throughout the night.

On PD "Sleep disorders", they are the consequence of the next level of the chain "Stress-Anxiety-Sleep disorders" by the following reasons:

a) PD patients reports "disturbed sleep patterns" long before motor dysfunction [10].

b) PD patients are affecting their "quality of life" and presents many aspects of "cognition deterioration" [12].

c) PD patients presents "NREM sleep instability" even in earlier stages [11].

d) PD patients have "Rapid Eye Movement (REM) sleep behavior disorder (RBD) ", that is characterized by dream enactment behavior [15], frequently reporting: flailing arms, leaping from bed, crawling or running [30].

e) PD patients reports many others physical and cognitive deterioration [1]

"Neural damage" is the stage where the response of the normal brain cells is altered. PD is caused by the impairment or "death of dopamine-produced in nerve cells (neurons) in a region of the brain called the substantia nigra", which controls the body's balance and movement, with a big probably cause by "sleep disorders" that didn't allow the need of clearing damaged proteins and waste from the brain cells in many neural pathways" [31]

As shown in Table 1, the "dopamine" is a chemical that acts as a messenger transmitting nerve signals from one neuron to another in the brain to control body movements as indicate in Figure 1c. It is also involved in behavior and cognition, memory and learning, sleep, and mood. The loss of these "neurotransmitter in the neurons" by the results of sequences of "Stress-Anxiety-Sleep disorders-neural cyclic chain", alter the production of many "neurotransmitters" that are reflected as "Parkinson's disease symptoms". the decreased specially on "dopamine", lead to abnormal brain activity and the symptoms of the PD such as: abnormal muscle movements, cognitive impairment and others PD symptoms. Neuropathological studies demonstrate significant damage in brain regions and their causes as stated in the following research reports:

a. In the research paper from: William P. Flavin et.al.,
"Endocytic vesicle rupture is a conserved mechanism of cellular invasion by amyloid proteins" [32,33]. Neurodegenerative diseases are caused by the death of neurons and other cells in the brain", with different diseases affecting different regions of the brain by "amyloid proteins" that are folded abnormally form clumps inside brain cells. Where:

a) "Parkinson's disease affects movement and cognition" thru "accumulation of alpha-synuclein protein".

b) "Alzheimer's disease destroys memory" thru "accumulation of amyloid-beta and tau protein"

c) "Huntington's disease affects movement and cognition" thru "accumulation of huntingtin protein"

All three diseases are progressive, debilitating and incurable. Previous research has suggested that in all three diseases these clumps spread from cell to cell, eventually leading to cell deaths.

b. In the research paper from: J. Swick: "Parkinson's disease and sleep/wake disturbances" [8]. The symptoms of Parkinson's disease usually appear when about 70-80 percent of nerve cells in the "substantia nigra" have been lost [34]. It is thought that a combination of genetic, and environmental factors contributes to the development of the disease.

c. In the research paper from: Nicolaas I. Bohnen et al. "Sleep Disturbance as Potential Risk and Progression Factor for Parkinson's Disease" [31]: "Sleep behavior disorder (RBD)" is common among the $\alpha$-synucleinopathy disorders of PD, dementia with Lewy bodies (DLB) and Multiple System Atrophy (MSA) probably because in these diseases cell loss is common within neuronal structures regulating Rapid Eye Movement (REM), that is an sleep disturbance" [35].

d. In the research paper from: Eti Ben Simon et al. "Overanxious and under-slept" [29]. "Deep sleep" restores the "medial prefrontal cortex" mechanisms that restore "emotion". This lowers emotional and physiological reactivity, "preventing the escalation of stress and anxiety". After a night of no sleep, brain scans showed a shutdown of the "medial prefrontal cortex", which normally helps keep our "anxiety" in check, while the brain's deeper emotional centers were overactive. "Our study strongly suggests that insufficient sleep amplifies levels of anxiety and, conversely, that deep sleep helps reduce such stress". "Deep sleep" seems to be a "natural anxiolytic (anxiety inhibitor)", so long as we get it each and every night"

e. In the research paper source from: University of Rochester 
Medical Center "Immune cells rewire and repair the brain while we sleep" [36]. Science tells us that a lot of good things happen in our brains while we sleep - "learning and memories are consolidated and waste is removed, among other things". New research shows for the first time that important immune cells called "microglia" - which play an important role in reorganizing the connections between nerve cells, fighting infections, and repairing damage are also primarily active while we sleep.

f. In the research paper from: Elena M Vazey \& Gary Aston-Jones, "The emerging role of norepinephrine in cognitive dysfunctions of Parkinson's disease" [37] "Parkinson's disease (PD) is the second most common neurodegenerative disorder", affecting $1 \%$ of the population over age 60 and $3 \%$ after 65 . In those patient's cognitive dysfunction is a persistent issue that impairs quality of life and productivity. Neuropathological studies demonstrate significant damage in brain regions outside the "nigral dopamine (DA) system", including early degeneration of "locus coeruleus norepinephrine (LC$\mathrm{NE}$ ) neurons", yet discussion of PD and "treatment focus has remained dopaminergic-based". "Motor symptoms benefit from DA replacement for many years, but other symptoms including several cognitive deficits continue unabated".

g. In the research paper from "Parkinson's news today: What Causes Parkinson's Disease?" [38]. "PD disease patients may have damaged nerve endings that produce norepinephrine", a signal messenger similar to "dopamine" that controls body functions such as pulse and blood pressure. This may explain some of the nonmotor symptoms of the disease, such as fatigue and blood pressure problems. It is thought that a combination of genetic and environmental factors contributes to the development of the disease.

h. In the research paper from Yuval Nir et al. "Selective neuronal lapses precede human cognitive lapses following sleep deprivation" [39]. During sleep deprivation, homeostatic and circadian processes interact to build up sleep pressure, which results in slow behavioral performance (cognitive lapses) typically attributed to attentional thalamic and frontoparietal circuits. During cognitive lapses, Local Field Potentials (LFP) exhibit a relative local increase in slow/theta activity that is correlated with degraded single-neuron responses and with baseline theta activity. Our results show that cognitive lapses involve local state-dependent changes in neuronal activity already present in the "medial temporal lobe (MTL)".

i. In the research paper from: Brandon E Hauer et al. "The Reuniens Nucleus of the Thalamus Has an ssential Role in Coordinating Slow-Wave Activity between Neocortex and Hippocampus" [40]. The" nucleus reuniens" is responsible for coordinating synchronous slow waves between the prefrontal cortex and hippocampus during sleep. The findings suggest the "nucleus reuniens" plays a critical role in sleep-dependent memory consolidation as a "Circuit responsible for building memories during sleep".

\section{Theory of Stress-Anxiety-Sleep Disorders-Neural Damage Cyclic chain and the Progression of Parkinson's \\ Disease}

As mention and explain, hundreds of research publications around the world are obtaining more and more results to conclude or suggest in some way: "The Theory of Stress-Anxiety-Sleep disorders-neural damage cyclic chain and the progression of Parkinson's disease" that can be explain as shown in Table 2 .

\begin{tabular}{|c|l|}
\hline Table 2: The proposed Theory of Stress-Anxiety-Sleep Disorders-Neural Damage cyclic chain and the progression of Parkinson's disease. \\
\hline Observations & $\begin{array}{l}\text { When the "synapse chemical activity" on many "neurons synapses" in the "neural pathway" is affected in some degree for factors as: } \\
\text { "Stress-Anxiety-Sleep disorders" and factor as genetics and others, then the release of "neurotransmitters" as: "dopamine, "GABA", and } \\
\text { many others, the release of "neurotransmitters" are diminished or stopped. }\end{array}$ \\
\hline 1 & $\begin{array}{l}\text { By consequence of the diminished "neurotransmitters", many normal human communication body functions will have problems in } \\
\text { their trajectories using the "neural pathways" as: orders to move muscles or other human organs as presented in "motor pathways", } \\
\text { and/or the reception of information in the brain as smell signals or any other human senses signals that are using "sensory pathways", } \\
\text { and/or affecting "neurotransmitters pathways" that are reflected on diminished human cognition. }\end{array}$ \\
\hline 2 & $\begin{array}{l}\text { These types of trajectories with body signals obstacles on "neural pathways" identified as "neuronal damage", that are in charge of the } \\
\text { necessary communication for the human body functions by the brain are reflected as "Parkinsonism symptoms", that include: "primary } \\
\text { motors" that are directly related to movement, "secondary motors" that are consequence of movement disorders, and "non-motors" } \\
\text { that are not related to movement disorders. }\end{array}$ \\
\hline 3 & $\begin{array}{l}\text { "Stress-Anxiety-Sleep Disorders-Neural damage", these factors are related and they are repetitive with behavior as a kind of cyclic } \\
\text { chain, where the initial factor could be anyone of them and grow a little more the intensity on each loop affecting the "progression of } \\
\text { Parkinson' disease", where: }\end{array}$ \\
\hline 4 &
\end{tabular}




\begin{tabular}{|c|c|}
\hline a. & $\begin{array}{l}\text { "Stress "is very frequently but no necessary the first stage, defined as the natural body's reaction from PD patients to a challenge or } \\
\text { demand in their actions that has been limited by any physical, cognitive diminished for the neurological disease. That also, it could come } \\
\text { from any event or thoughts that increase frustration, angry, pain or nervousness. The "Stress" can indirectly alter the synapse chemical } \\
\text { activity in neurons" [14,32]. }\end{array}$ \\
\hline b. & $\begin{array}{l}\text { "Anxiety" as the normal reaction to "stress". Where "PD Anxiety" is more noticeable with physical effects and mental situation on the } \\
\text { next level as: difficulty of controlling worries, exaggerated feeling for sudden shock or alarm that producing physical and cognitive } \\
\text { effects that can take them to deeper levels like "depression" and generate more cognitive declinations. "Anxiety" can indirectly alter } \\
\text { even more the synapse chemical activity in neurons" }[1,2,27] \text {. }\end{array}$ \\
\hline c. & $\begin{array}{l}\text { "Sleep disorders" are based in sleep changes due to multiple factors, on PD patients mainly are reactions to: "stress"," anxiety", and } \\
\text { "declination on the overall health including pain, desperation as sudden sensation of fear and others". "sleep deprivation disrupts } \\
\text { in the PD brain cells, the ability to communicate with each other, leading to temporary mental lapses that affect memory and visual } \\
\text { perception, leading for cognitive lapses in how to perceive the world and their reactions". Definitively "Sleep disorders" affect the } \\
\text { chemical activities and release of the neurotransmitters in synapses" }[2,4,5,8,9,10,11,12,13,19,24,30,31,34] \text {. }\end{array}$ \\
\hline d. & $\begin{array}{l}\text { "Neural damage" is the stage where the response of the normal brain cell is "altered to the limitation in the release of many necessary" } \\
\text { neurotransmitters during the synapses". PD is caused mainly by the "impairment or death of many neurotransmitters, with specially } \\
\text { emphasis in the dopamine-produced in nerve cells (neurons) in a region of the brain called the substantia nigra", which controls the } \\
\text { body's balance and movement, with a big probably caused by genetic alterations or sleep disorders that didn't allow the "need of } \\
\text { clearing damaged proteins and waste from the brain cells in many neural pathways" [30]. "and also affecting cognition by avoiding the } \\
\text { memory consolidation needed during a deep sleep" }[19,28,29,30] \text {. }\end{array}$ \\
\hline e. & $\begin{array}{l}\text { After the "Stress-Anxiety-Sleep Disorders-Neural Damage chain in PD patients" is completed, initiated in any step by many external or } \\
\text { internal reasons, the chain is reinitiated to a higher level forming a bigger cyclic chain as shown in figure } 2 \text { represented with dash circles } \\
\text { indicating the progression in Parkinson's disease. }\end{array}$ \\
\hline 5 & $\begin{array}{l}\text { The proposed, "Theory of Stress-Anxiety-Sleep Disorders-Neural Damage cyclic chain and the progression of Parkinson's disease" is } \\
\text { repeated undefinedly worsening the symptoms of Parkinson's disease that are only detected after 70-80 percent of nerve cells in the } \\
\text { "substantia nigra" have been lost }[8,37] \text {. }\end{array}$ \\
\hline
\end{tabular}

Recommendations to Implement the "The Theory of Stress-Anxiety-Sleep Disorders-Neural Damage Cyclic Chain and the Progression of Parkinson's Disease".

Based on "The Theory of Stress-Anxiety-Sleep Disorders-Neural Damage cyclic chain and the progression of Parkinson's disease " shown in Figure 2, explained in the last section, stated in Table
2, and taking into account that Parkinson's doesn't affect only the person diagnosed with the disease; it can impact loves ones, family members, friends and caregivers too. I can deduct the following "7 more important recommendations for PD patients and their caregivers" for a good implementation of the theory and achieve the decreased progression of this neurological disease. The basic recommendations are indicated in the following Table 3.

Table 3: The "7 most important recommendations for PD patients and their caregivers" for a successful implementation of "The Theory of StressAnxiety-Sleep Disorders-Neural Damage cyclic chain and the progression of Parkinson's disease".

\begin{tabular}{|c|c|}
\hline $\begin{array}{l}\text { Recommendation for } \\
\text { PD patients and } \\
\text { caregivers }\end{array}$ & Reasons for the recommendation \\
\hline $\begin{array}{l}\text { 1) "Love and comprehension } \\
\text { to them" }\end{array}$ & $\begin{array}{l}\text { "Parkinson's disease it's not about a big fight with the disease, it is about "acceptation with love and comprehension of } \\
\text { the reality that PD patients have a neurological progressive disease", } \\
\text { "Recognize that: stress, anxiety and others kinds of fear attitudes make the PD symptoms worse". } \\
\text { "It is time to slow down. "Take life one day at a time!" } \\
\text { "Each day is different for PD patients" } \\
\text { "Accept the fact that there are good days, and bad days". } \\
\text { "Every day count your blessings; you will find many more than you knew, be proud and grateful for them". }\end{array}$ \\
\hline 2) "Eat healthy" & $\begin{array}{l}\text { "PD patients need good energy from real food! say goodbye to junk food" } \\
\text { "Grease, fast food and proteins make harder to your body to digest and absorbs your PD medications". } \\
\text { "Eat Healthy food based on fruits grains and vegetables, fish with omega 3, poultry and eat little red meat". }\end{array}$ \\
\hline 3) "Stress and Anxiety handle" & $\begin{array}{l}\text { "Recognize that all PD patients need incremental help from others as: family members, friends, take givers, medical } \\
\text { doctors, and that there are many therapies can help you to handle the "stress and anxiety", } \\
\text { "Write down your negative thoughts and ask if they are true, because root in stresses and anxiety mind can be distort } \\
\text { the severity of the real situation, and analyze if it is possible to take back control of them, if not don't worry time will } \\
\text { say how" } \\
\text { "Forget multitasking and multiprocessing, PD patients must be concentrated in doing and thinking only one thing at } \\
\text { the time to avoid unnecessary stress" } \\
\text { "Maintain as possible positive attitude with good humor as frequently as possible". }\end{array}$ \\
\hline
\end{tabular}




\begin{tabular}{|c|l|}
\hline & $\begin{array}{l}\text { "Recognize that deep sleep is something necessary for your mind and body specially in PD patients to control the } \\
\text { progression of the disease!" } \\
\text { "Try first home remedies and relaxing techniques to sleep", } \\
\text { 4) "Sleep well" } \\
\text { "Gives thanks every day for all blessing received thought your life". } \\
\text { "Sleep disturbances, it is very common for PD patients; don't' be afraid to ask medical help support if you cannot } \\
\text { handle your daily situations and tasks due to sleep deprivation" }\end{array}$ \\
\hline 5) "Exercise body and mind" & $\begin{array}{l}\text { "Moderate daily exercise according to the disease progression", exercise releases feel-good chemicals that are necessary } \\
\text { in your brain", } \\
\text { "do mind games and keeping learning thing to boost the creation of new neural pathways in your brain". }\end{array}$ \\
\hline $\begin{array}{c}\text { 6) "Medicines always in } \\
\text { time and keep a record of } \\
\text { their intakes, specially the } \\
\text { combination of medicines" }\end{array}$ & $\begin{array}{l}\text { "Keeping a record of medicines intake with the purpose of detect on time side effects, register any change observed, } \\
\text { time off /time off, each fall and why happen and how can be avoided, analyze them frequently and give the feedback to } \\
\text { your healthcare team and neurologist". } \\
\text { "Never add new medicines/suddenly increment / decrement or discontinue the doses of these PD medications without } \\
\text { supervision from your healthcare team and your neurologist". }\end{array}$ \\
\hline 7) "Socialize and stay busy" & $\begin{array}{l}\text { "Isolation never help with PD", } \\
\text { "Be involved in any conversation around, everybody learns from each other!" } \\
\text { "Always thing in other's needs " } \\
\text { "Maintain up to date on advances on PD new treatments" } \\
\text { "Stay busy according to the level of the disease" } \\
\text { Do that special easy things that recomfort you as: listen to music, coloring book, drawing, hobbies, gardens and nature } \\
\text { activities, watching movies, browsing internet, cards game, and others", }\end{array}$ \\
\hline
\end{tabular}

\section{Conclusion}

Parkinson' disease is a neurologic disease that affect the functionality if the brain modifying the neural connectivity due to cell death. All actual medication and procedures available today only help to improve the quality of life of the patients. "The Theory of Stress-Anxiety-Sleep Disorders-Neural Damage cyclic chain and the progression of Parkinson's disease "and their deducted prevention actions explained as "The 7 most important recommendations for PD patients and their caregivers" based on it, to try to help in the decrease of the progression of Parkinson's disease.

There are many more issues to resolve on the development of these disease that affect PD patients. There are necessity for new biomarkers, and new methods for early detection on Parkinson's disease, new techniques for follow-up the progression to achieve more understanding of this neurological disease and others. We need to know the real PD causes, where PD begins and why, find new biomarkers to measure the damage level from early stages, develop more stable and effective PD medications, new PD surgical treatment, better mathematical tools and smart computer algorithms for the correct analysis, diagnostic and prescription and many mores issues to understand and resolve the issues of this highly varied disease. We need more from you: Please share your information about your PD journey, possible causes that you believe, about your progression, all your symptoms, and many things, sent that information to PD researches like: garzaulloa.org, michaeljfox. org, parknison.org and other PD research organizations. Don't forget to participate, if it is possible in clinicals trials evaluation to help researchers to find a possible cure" $[43,44]$.

Note*: I hardly recommend to read more PD information about: stages, symptoms, types, treatments, medications, surgical treatments, complementary/alternative therapies, factors, PD journeys feedback, etc. on my free download papers: "Update on Parkinson's Disease" [1], and "A Parkinson's Disease Journey from Patient side view" [41], and buy my specialized books on "motor symptoms analysis of neurological diseases published in 2018": "Applied Biomechatronic Using Mathematical Models"[42], and future book to be publish in 2021: "non-motors analysis of neurological diseases":" Applied Biomedical Engineering Using Artificial Intelligence And Cognitive Models".

\section{Dr. Jorge Garza-Ulloa}

jorge@garzaulloa.org,www.garzaulloa.org

El Paso, Texas USA/Cd. Juárez, Chih, Mexico..

\section{References}

1. Jorge Garza-Ulloa (2019) Update on Parkinson's Disease. Am J Biomed Sci \& Res 2(6): 229-236.

2. Rosanna Palmeri, Viviana Lo Buono, Lilla Bonanno, Chiara Sorbera, Vincenzo Cimino, et al. Potential predictors of quality of life in Parkinson's Disease: Sleep and mood disorders, Journal of Clinical Neuroscience 70: 113-117.

3. https://www.sleep.org/articles/what-happens-during-sleep/

4. Aline Duarte Folle, Kimberly C Paul, Jeff M Bronstein, Adrienne M Keener, Beate Ritz (2019) Clinical progression in Parkinson's disease with features of REM sleep behavior disorder: A population-based longitudinal study, Parkinsonism \& Related Disorders 62: 105-111.

5. https://adaa.org/understanding-anxiety/related-illnesses/sleepdisorders

6. (2019) REM sleep behavior disorder.

7. Jorge Garza-Ulloa (2018) Introduction to human neuromusculoskeletal systems. Applied Biomechatronics using Mathematical Models, Academic Press 53-118.

8. Suzuki K, Miyamoto M, Miyamoto T, Hirata K (2015) Parkinson's disease 
and sleep/wake disturbances, Curr Neurol Neurosci Rep 15(3): 8.

9. J Andrew Albers, Pratap Chand, A Michael Anch (2017) Multifactorial sleep disturbance in Parkinson's disease, Sleep Medicine 35: 41-48.

10. Jorge S Valadas, Giovanni Esposito, Dirk Vandekerkhove, Katarzyna Miskiewicz, Liesbeth Deaulmerie, et al. (2018) ER Lipid Defects in Neuropeptidergic Neurons Impair Sleep Patterns in Parkinson's Disease. Neuron 98(6): 1155-1169.

11. Lorenzo Priano, Matteo Bigoni, Giovanni Albani, Luigi Sellitti, Emanuela Giacomotti, et al. (2019) Sleep microstructure in Parkinson's disease: cycling alternating pattern (CAP) as a sensitive marker of early NREM sleep instability. Sleep Medicine 61: 57-62.

12. James E Delorme, Varna Kodoth, Sara J Aton (2019) Sleep loss disrupts Arc expression in dentate gyrus neurons, Neurobiology of Learning and Memory 160: 73-82.

13. Stavros J Baloyannis, Vassiliki Costa, Ioannis S Baloyannis (2006) Morphological alterations of the synapses in the locus coeruleus in Parkinson's disease, Journal of the Neurological Sciences 248(1-2): 3541.

14. Rosanna Palmeri, Viviana Lo Buono, Lilla Bonanno, Chiara Sorbera, Vincenzo Cimino, et al. Potential predictors of quality of life in Parkinson's Disease: Sleep and mood disorders, Journal of Clinical Neuroscience 70: 113-117.

15. Aline Duarte Folle, Kimberly C Paul, Jeff M Bronstein, Adrienne M Keener, Beate Ritz (2019) Clinical progression in Parkinson's disease with features of REM sleep behavior disorder: A population-based longitudinal study, Parkinsonism \& Related Disorders, Volume 62: 105111.

16. https://www.epda.eu.com/about-parkinsons/diagnosis/parkinsonsprogression/

17. Jorge Garza-Ulloa (2018) Introduction to biomechatronics/biomedical engineering. Applied Biomechatronics using Mathematical Models, Academic Press pp. 1-51

18. Shreya Bhat U, Rajendra Acharya, Yuki Hagiwara, Nahid Dadmehr, Hojjat Adeli (2018) Parkinson's disease: Cause factors, measurable indicators, and early diagnosis, Computers in Biology and Medicine 102: 234-241.

19. A Bjorklund, JH Kordower (2013) Cell therapy for Parkinson's disease: what next? Mov Disord 28(1): 110-115.

20. Himadri Shekhaar Baul, Ceera Manikandan, Dwaipayan Sen (2019) Cannabinoid receptor as a potential therapeutic target for Parkinson's Disease. Brain Research Bulletin 146: 244-252.

21. https://parkinsonsnewstoday.com/what-causes-parkinsons-disease/

22. Ziv Gan-Or, Roy N Alcalay, Guy A Rouleau, Ronald B Postuma (2018) Sleep disorders and Parkinson disease; lessons from genetics, Sleep Medicine Reviews 41: 101-112.

23. Shreya Bhat U, Rajendra Acharya, Yuki Hagiwara, Nahid Dadmehr, Hojjat Adeli (2018) Parkinson's disease: Cause factors, measurable indicators, and early diagnosis, Computers in Biology and Medicine 102: 234-241.

24. CM Tanner (2011) Environmental factors and Parkinson's: what have we learned? Neurology 39(5): 660-664.

25. J Campdelacreu (2014) Parkinson's disease and Alzheimer disease: environmental risk factors. Neurologia 29(9): 541-549.

26. https://www.epda.eu.com/about-parkinsons/diagnosis/parkinsonsprogression/
27. Melanie A Horning, Ju Young Shin, Leigh Ann DiFusco, Michelle Norton, Barbara Habermann (2019) Symptom progression in advanced Parkinson's disease: Dyadic perspectives, Applied Nursing Research 151193.

28. https://neurosciencenews.com/immune-cells-rewire-repair-15094/

29. Eti Ben Simon, Aubrey Rossi, Allison G Harvey, Matthew P Walker (2019) "Overanxious and underslept". Nature Human Behaviour.

30.http://sleepeducation.org/news/2011/12/15/calling-dreamenactment-behavior-back-to-bed

31. Nicolaas I Bohnen, Michele TM Hu (2019) Sleep Disturbance as Potential Risk and Progression Factor for Parkinson's Disease, J Parkinsons Dis 9(3): 603-614.

32. Rianne D Stowell, Grayson O Sipe, Ryan P Dawes, Hanna N Batchelor, Katheryn A Lordy, et al. "Noradrenergic signaling in the wakeful state inhibits microglial surveillance and synaptic plasticity in the mouse visual cortex". Nature Neuroscience 22: 1782-1792.

33. William P Flavin, Luc Bousset, Zachary C Green, Yaping Chu, Stratos Skarpathiotis, et al. "Endocytic vesicle rupture is a conserved mechanism of cellular invasion by amyloid proteins". in Acta Neuropathologica 134(4): 629-653

34. JL George, S Mok, D Moses, S Wilkins, AI Bush, RA Cherny, et al. (2009) Targeting the Progression of Parkinson's Disease, Curr Neuropharmacol $7(1): 9-36$.

35. Stuart J McCarter, Erik K St Louis, and Bradley F Boeve (2012) REM Sleep Behavior Disorder and REM Sleep Without Atonia as an Early Manifestation of Degenerative Neurological Disease, Curr Neurol Neurosci Rep (2): 182-192.

36. (2019) Immune cells rewire, repair brain while we sleep: Study.

37. Elena M. Vazey, Gary Aston-Jones (2012) The emerging role of norepinephrine in cognitive dysfunctions of Parkinson's disease, PMC Front Behav Neurosci 6: 48.

38. https://parkinsonsnewstoday.com/what-causes-parkinsons-disease/

39. Yuval Nir, Thomas Andrillon, Amit Marmelshtein, Nanthia Suthana, Chiara Cirelli, Giulio Tononi \& Itzhak Fried. Selective neuronal lapses precede human cognitive lapses following sleep deprivation, Nature Publishing Group 23: 1474-1480.

40. Brandon E Hauer, Silvia Pagliardini, Clayton T Dickson (2019) "The Reuniens Nucleus of the Thalamus Has an Essential Role in Coordinating Slow-Wave Activity between Neocortex and Hippocampus". eNeuro 17: $6(5)$

41. Jorge Garza-Ulloa (2019) A Parkinson's Disease Journey from Patient side view. Am J Biomed Sci \& Res 5(1): 25-32.

42. Jorge Garza-Ulloa (2018) Applied Biomechatronics using Mathematical Models.

43. Rianne D Stowell, Grayson O Sipe, Ryan P Dawes, Hanna N Batchelor Katheryn A Lordy, et al. "Noradrenergic signaling in the wakeful state inhibits microglial surveillance and synaptic plasticity in the mouse visual cortex". Nature Neuroscience.

44. https://parkinsonsnewstoday.com/what-is-parkinsons-disease/

45. Velma TE, et al. (2019) Gut microbiota in Parkinson's disease: Temporal stability and relations to disease progression,EBioMedicine 44: 691707. 